\title{
Peculiarities of phase transformations in SiC crystals and thin films with in-grown original defects
}

\author{
S.I. Vlaskina ${ }^{1}$, G.N. Mishinova ${ }^{2}$, V.E. Rodionov ${ }^{1}$, G.S. Svechnikov ${ }^{1}$ \\ ${ }^{1}$ V. Lashkaryov Institute of Semiconductor Physics, National Academy of Science of Ukraine, \\ 41, prospect Nauky,03028 Kyiv, Ukraine; e-mail: businkaa@mail.ru \\ ${ }^{2}$ Taras Shevchenko Kyiv National University, 64, Volodymyrs'ka str., 01033 Kyiv, Ukraine
}

\begin{abstract}
Phase transformations of $\mathrm{SiC}$ crystals and thin films with in-grown original defects have been studied. The analysis of absorption, excitation and low-temperature photoluminescence spectra testifies to formation of new micro-phases during the growth. The complex spectra can be decomposed into similar structure-constituting spectra shifted against each other on the energy scale. These spectra are indicative of formation of new nanophases. Taking into account the position of the short-wave edge in the zerophonon part of the SF-i spectra as well as the position of corresponding excitation spectra and placing them on the well-known linear dependence of the exciton gap $\left(E_{g x}\right)$ on the percentage of hexagonally in different polytypic structures, one can obtain a hint to the percentage of hexagonally in the new metastable structures appearing in the $6 \mathrm{H}(33)$ matrix or in the growth process. The SF spectra are indicative of the appearance of these metastable structures.
\end{abstract}

Keywords: phase transformation, absorption spectrum, excitation spectrum, lowtemperature photoluminescence spectrum, polytypic structure, $\mathrm{SiC}$ crystal.

Manuscript received 11.07.14; revised version received 26.08.14; accepted for publication 29.10.14; published online 10.11.14.

\section{Introduction}

The correlations between the crystal structure and optical properties (photoluminescence, absorption) are under discussion for polytypic materials. Silicon carbide polytypes are the best choice for these investigations. Actual atomic structures, the accompanying lattice vibrations, properties of layered combinations of polytypes can be considered from the optical spectra.

In this work, we report phase transformations of $\mathrm{SiC}$ crystals and thin films with in-grown original defects. SiC crystals were grown applying the Tairov method, films were obtained using the "sandwich"method and chemical vapor deposition.

The analysis of absorption, excitation and lowtemperature photoluminescence spectra testifies to formation of new microphases during the growth. The complex spectra can be decomposed into similar structure-constituting spectra shifted against each other on the energy scale. These spectra are indicative of formation of new nanophases.

Joint consideration of photoluminescence spectra, excitation photoluminescence spectra and absorption spectra testifies to the uniformity of different spectra and the autonomy of each of them. Structurally, the total complex spectra are correlated with the crystal imperfection and the peculiarities of one-dimensional disorder. Three different types of spectra have three different principles of construction and behavior.

Nanostructure-indicator's spectra are placed on the wide donor-acceptor pair (DAP) spectrum in crystals and films in case of higher concentrations of non- 
compensated impurities. Spectra of excitation, photoluminescence and absorption spectra indicate formation of nanostructures $\mathrm{SiC}(8 \mathrm{H}-, 10 \mathrm{H}-, 14 \mathrm{H}-\mathrm{SiC})$.

In this paper, the D-layers with in-grown disordering in $\alpha$-SiC crystals and films (mainly $6 \mathrm{H}-\mathrm{SiC}$ ) as well as zones of $\beta \rightarrow \alpha$ solid-phase transformations were investigated. Low-temperature photoluminescence (LTPL) spectroscopy was used as a highly sensitive and accurate method to the structure changes [1-3].

\section{Experimental}

LTPL spectra were registered by the DFS-12 spectrograph with the photodetector (FEU-79). In photoluminescence (PL) experiments a nitrogen LGI-21 $(337 \mathrm{~nm}, \quad 3.68 \mathrm{eV})$ or helium-cadmium LG-70 $(441.6 \mathrm{~nm}, 2.807 \mathrm{eV})$ laser were used. Also a mercury ultrahigh pressure lamp SVDSh-1000 with the UV-2 filter, and a xenon lamp DKSSh-1000 were used.

The group of monocrystalline $\alpha-\mathrm{SiC}$ crystals grown using Lely's (Tairov's) method under consideration were separated by three groups:

1. $N_{S F}$-samples (colourless): pure crystals with the impurity concentration of $N_{D}-N_{A} \sim(6 \ldots 7) \cdot 10^{16} \mathrm{~cm}^{-3}$, $N_{D} \sim(7 \ldots 8) \cdot 10^{16} \mathrm{~cm}^{-3}, N_{A}<1 \cdot 10^{16} \mathrm{~cm}^{-3}$.

2. $N_{D L}$-samples (doped, light-green): with $N_{D}-N_{A}$ $\sim(2 \ldots 8) \cdot 10^{16} \mathrm{~cm}^{-3}, N_{D} \sim(5 \ldots 8) \cdot 10^{17} \mathrm{~cm}^{-3}$, and $N_{D}-N_{A}$ $>3 \cdot 10^{17} \mathrm{~cm}^{-3}, N_{D}>1 \cdot 10^{18} \mathrm{~cm}^{-3}$.

3. Cubic crystals of $N_{\beta}$-samples of the $n$-type with $N_{D} \leq 1 \cdot 10^{17} \mathrm{~cm}^{-3}$ (light yellow). Undoped $\mathrm{SiC}$ single crystals with the impurity concentration of $N_{D}-N_{A} \sim$ $(2 \ldots 8) \cdot 10^{16} \mathrm{~cm}^{-3}, N_{A} \sim(2 \ldots 8) \cdot 10^{17} \mathrm{~cm}^{-3}$, and $N_{D}-N_{A} \sim$ $(1 \ldots 5) \cdot 10^{17} \mathrm{~cm}^{-3}, N_{D} \geq 1 \cdot 10^{18} \mathrm{~cm}^{-3}$ were investigated.

Films were prepared using the sublimation "sandwich" and CVD methods.

\section{Results and discussion}

Fig. 1 shows LTPL spectra according to structural imperfection and the impurity concentration in $\alpha-\mathrm{SiC}$ crystals. Structurally perfect $6 \mathrm{H}-\mathrm{SiC}$ crystals (or perfect blocks of the crystal which coherently coalesce with disordering layers) show a typical spectrum of nitrogenbound exciton complexes (PRS) together with the linear $\mathrm{ABC}$-spectrum related to $\mathrm{Ti}[(\delta-0)-(\mathrm{a})]$ and emission spectra of the donor-acceptor pairs.

Topical for this paper are the spectra of $[(\delta-\mathrm{I})-$ (a)], [( $\delta$-II $)-(\mathrm{a})] N_{S F}[4-6]$ as well as $[(\delta-\mathrm{I})-(\mathrm{b})],[(\delta-\mathrm{II})$ - (b)] $N_{D L}$ samples [7,8].

The peculiarity of the photoluminescence spectra related to the zone of disorder to a great extent depends on the impurity concentration in the matrix as a whole. In pure samples $N_{S F}$ (case (a) $i=1,2,3, \ldots$ ) at low temperatures the SF-i spectra are dominant, whereas the intensity of the DL-i spectra is very low. On the contrary, in the doped samples the DL-i spectra are dominant and located on a broad DAP emission band, while SF-i spectra are practically invisible.

Peculiarities of the Laue patterns of the D-layers in $6 \mathrm{H}-\mathrm{SiC}$ crystals are the same as for the $3 \mathrm{C}-\mathrm{SiC}$ crystals after phase transformation [9]. Pure samples with the SF-i and DL-i series, $N_{D}-N_{A} \sim(2 \ldots 8) \cdot 10^{16} \mathrm{~cm}^{-3}$, $N_{A} \sim(2 \ldots 8) \cdot 10^{17} \mathrm{~cm}^{-3}$ and samples with only DL-i spectra, $N_{D}-N_{A} \sim(2 \ldots 7) \cdot 10^{17} \mathrm{~cm}^{-3}, N_{D}<1 \cdot 10^{18} \mathrm{~cm}^{-3}$ show a linear dependence of intensity of the DL-i spectra on the impurity concentration [9].

For the absorption spectra of DL samples [9], it was shown that absorption is spread far to the lower energy region relative to $E_{g x}^{6 H}$ (exciton band gap in $6 \mathrm{H}$ structure). This can serve as an evidence of formation of structures with a higher percentage of cubic $\alpha$-SiC than $6 \mathrm{H}-\mathrm{SiC}$

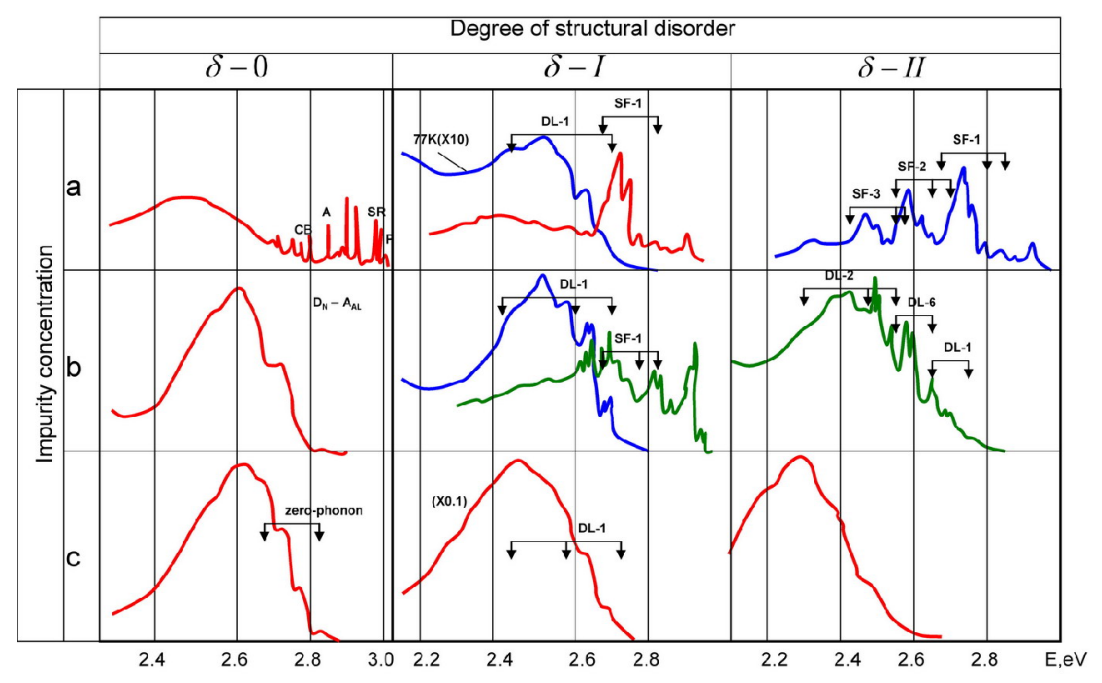

Fig. 1. LTPL spectra according to the structural imperfection and impurity concentration in $\alpha$-SiC crystals. 
General panorama of the PL spectra of the samples consists of a certain set of the SF-spectra with different intensities. Sometimes, it is peculiar to the different parts of the same crystal. It was shown that all the SF-spectra have the identical character, principal of structure, independent of the position on the energy scale.

Decoding the SF-spectrum (as an example the most frequent SF-1 spectrum) shows that the total structure of the spectrum can be obtained by additive summation of the phonon replica of some zero-phonon parts. In this case, phonons of the edge of the extended Brillouin zone of $\mathrm{SiC}$ (TA - $46 \mathrm{meV}, \mathrm{LA}-77 \mathrm{meV}$, TO - $95 \mathrm{meV}$, LO $-104 \mathrm{meV}$ ) were involved. While the zero-phonon part itself is not detected, it was "redesigned" according to the structure of the TA replica and transferred as a whole by the TA phonon energy $46 \mathrm{meV}$ to the high energy region. Thin linear structure and its readability in case of different D-layers is different. The minimum halfwidth of this structure is about $1.5 \mathrm{meV}$. After defining the position of the zero-phonon part in the SF-1 spectrum on the energy scale, it appears that the short-wave part of the spectrum coincides with the position of the exciton band gap of $21 \mathrm{R}$ polytype at $T=4.2 \mathrm{~K}(2.853 \mathrm{eV})$.

Investigating the photoluminescence excitation spectra $\left(\eta_{i}\right)$ reveals that each SF-1 spectrum has its own excitation spectrum [6]. This may be the evidence of some kind of autonomy of the SF-1 spectrum, its independence from the special part of crystal disorder, and the explanation of their different contributions to the total spectra. It was shown that the long-wave edge of each $\eta_{i}$ spectrum coincides with the short-wave edge of the zero-phonon part of corresponding SF-1 spectrum. For the given sample, the overall excitation spectrum $\left(\sum \eta_{i}\right)$ corresponds to the variation of the absorption coefficient in the D-layer [2].

Consequently, it is possible by choosing suitable photon energy of the exciting radiation to completely eliminate the more short-wave DL-i spectra from the total complex panorama of the spectrum, i.e. resolve it into the independent components (Fig. 2).

We found that SF-1 and SF-2 spectra appeared under excitation by using the $\operatorname{Ar}^{+}$laser $(h v=2.54 \mathrm{eV})$, while these spectra are in the higher energy region. It may be due to the two-photon excitation with transfer of carriers to the higher energy bands through the intermediate states. The identical character of the SF-i spectra is backed up by the identical temperature behavior and peculiarity of this behavior made it possible to decode the thin structure of the spectra [6].

Taking into account the position of the short-wave edge of the zero-phonon part of the SF-i spectra: 2.853, $2.712,2.611, \ldots, 3.002 \mathrm{eV}$, i.e. SF-1, SF-2, SF-3, ..., SF-5, as well as the position of corresponding excitation spectra and placing them on the well known linear dependence of the exciton gap $\left(E_{g x}\right)$ on the percentage of hexagonally in different polytypic structures $[3,6]$ (Fig. 3), one can obtain a hint to the percentage of hexagonally of the new metastable structures appearing in the $6 \mathrm{H} \mathrm{(33)} \mathrm{matrix} \mathrm{or} \mathrm{in} \mathrm{the} \mathrm{growth} \mathrm{process.}$

$$
\begin{aligned}
& \text { SF- } 1-14 \mathrm{H}_{1}\langle 34\rangle-17.5 \AA-28.5 \%(\mathrm{~h}) \\
& \text { SF-2 }-10 \mathrm{H}_{2}\langle 55\rangle-25 \AA-20 \% \\
& \text { SF-3 - } 14 \mathrm{H}_{2}\langle 77\rangle-35 \AA-14.3 \%
\end{aligned}
$$

In several cases, the SF-5 spectra with the motive to build the quasi-polytype 33R (3332) - 37\% (h) were observed. Formation of the structures $10 \mathrm{H}_{2}\langle 55\rangle$ and $14 \mathrm{H}_{2}\langle 77\rangle$ but not $10 \mathrm{H}_{1}\langle 3223\rangle 40 \%$ and $14 \mathrm{H}_{1}\langle 4334\rangle$ (corresponds to $\langle 34\rangle$ in $21 \mathrm{R}$ ) with the same periods, but with the higher percentage of hexagonality is backed by the fact that exactly these structures are characterized by the percentage of hexagonality at which the correspondent values of $E_{g x}$ are pointed to.

The motive of construction $\langle 3223\rangle(40 \% \mathrm{~h})$ of the structure on the metastable microlevel corresponds to the motive of $15 \mathrm{R}$ polytype construction that occurs in a stable condition. The motive of $\langle 4334\rangle(28.6 \% \mathrm{~h})$ corresponds to the well-known stable $21 \mathrm{R}$ polytype.

Low-temperature photoluminescence spectra of pure $(\mathrm{Al}, \mathrm{N}) \alpha-\mathrm{SiC}$ crystals and pure $\beta$-SiC crystals (zones of $\beta \rightarrow \alpha$ solid-phase transformations) are represented by the same SF-i spectra, which are indicators of the intermediate metastable micro- and nanostructures with the medium intermediate percentage of hexagonality regarding $6 \mathrm{H}$ and $3 \mathrm{C}$ phases, namely $21 \mathrm{R}\langle 34\rangle, 10 \mathrm{H}_{2}\langle 55\rangle, 14 \mathrm{H}_{2}\langle 77\rangle$. In pure $\mathrm{SiC}$ crystals, the SF-i spectra are dominant [6]. In crystals with a higher impurity concentration, the DL-i spectra appear. The spectra of DL-i type are different from the SF-i spectra and have other principles of construction and behavior. They are located on a broad DAP emission band in crystals with higher concentrations of non-compensated impurities (Fig. 1). Structurally, the general complexity of the DL-i spectra correlated with the degree of disorder of the crystal and was related with the one-dimensional disorder, the same as in the case of the stacking fault (SF-i) spectra.

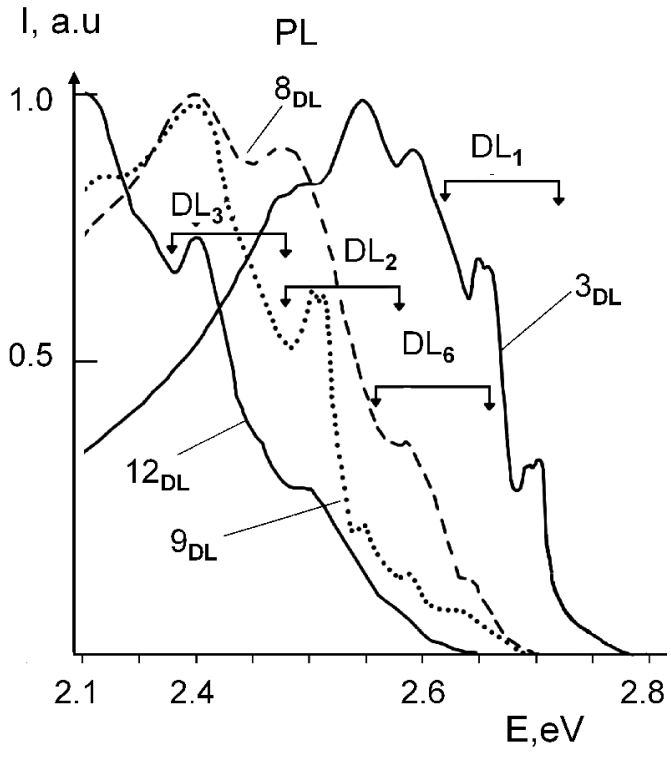

Fig. 2. DL-i spectra at $T=77 \mathrm{~K}$.

(C) 2014, V. Lashkaryov Institute of Semiconductor Physics, National Academy of Sciences of Ukraine 


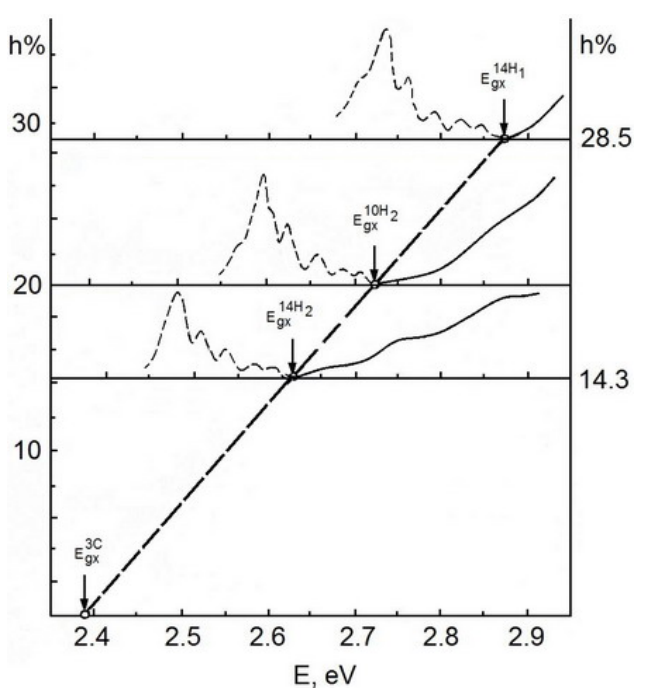

Fig. 3. SF-i spectrum allocation with respect to the linear dependence of the exciton gap $\left(E_{g x}\right)$ on the percentage of hexagonally to different polytypic structures. The long-wave edge of the excitation spectra is shown. Edge excitation spectra coincide with short edges of SF-i spectra at the point corresponding to definite values of $E_{g x}$.

The analysis of absorption, excitation and lowtemperature photoluminescence spectra suggest formation of a new microphase during the growth process and appearance of the deep level (DL) spectra. The complex spectra of the crystals can be decomposed into the so-called DL-i $(i=1,2,3,4)$ spectra. All the spectra of the DL type demonstrate identical behavior of the thin structure. If SF-i and DL-i spectra are reconciled at the long-wave part, this combination of spectra are along the line of the dependence $E_{g}=f$ (percentage of hexagonality). Herein, SF-i and DL-i spectra exist independently, they have different way to behave, however, match the same nanostructure transformations at $\alpha \rightarrow \beta$ and $\beta \rightarrow \alpha$ transformations [10-12].

\section{Conclusions}

The peculiarity of the photoluminescence spectra related to the zones of disorder to a great extent depends on the impurity concentration in the matrix as a whole. In pure samples, $N_{S F}$ (case (a) $I=1,2,3, \ldots$ ) at low temperatures the SF-i spectra are dominant, whereas the intensity of the DL-i spectra is very low. On the contrary, in the doped samples the DL-i spectra are dominant and located on a broad DAP emission band, while SF-i spectra are practically invisible.

Taking into account the position of the short-wave edge of the zero-phonon part in the SF-i spectra as well as the position of corresponding excitation spectra and placing them on the well-known linear dependence of the exciton gap $\left(E_{g x}\right)$ on the percentage of hexagonally in different polytypic structures, one can obtain a hint to the percentage of hexagonally of the new metastable structures appearing in the $6 \mathrm{H}(33)$ matrix or in the growth process. The SF spectra are indicative of the appearance of these metastable structures.

\section{References}

1. Fei Yan, Low Temperature Study on Defect Centers in Silicon Carbide, dissertation, University of Pittsburgh, 2009 (Dissertation LTPL-Choyke Pittsburg 2009, etd-08052009-012630).

2. I.S. Gorban and G.N. Mishinova, Basics of luminescent diagnostics of the dislocation structure of SiC crystals // Proc. SPIE, 3359, p. 187 (1998).

3. W.J. Choyke, H. Matsunami, G. Pensl, Silicon Carbide: Recent Major Advances. Springer, 2004.

4. S.I. Vlaskina, G.N. Mishinova, V.I. Vlaskin, V.E. Rodionov, G.S. Svechnikov, 6H-3C transformation in heated cubic silicon carbide 3C-SiC // Semiconductor Physics, Quantum Electronics and Optoelectronics, 14(4), p. 432-437 (2011).

5. S.I. Vlaskina, G.N. Mishinova, V.I. Vlaskin, G.S. Svechnikov, V.E. Rodionov, S.W. Lee, Silicon carbide phase transition in as-grown $3 \mathrm{C}-6 \mathrm{H}$ - polytypes junction // Semiconductor Physics, Quantum Electronics and Optoelectronics, 16(2), p. 132-136 (2013).

6. S.I. Vlaskina, G.N. Mishinova, V.I. Vlaskin, V.E. Rodionov, G.S. Svechnikov, 8H-, 10H-, 14H$\mathrm{SiC}$ formation in $6 \mathrm{H}-3 \mathrm{C}$ silicon carbide phase transitions // Semiconductor Physics, Quantum Electronics and Optoelectronics, 16(3), p. 272-278 (2013).

7. S.I. Vlaskina, D.H. Shin, $6 \mathrm{H}$ to $3 \mathrm{C}$ polytype transformation in silicon carbide // Jpn. J. Appl. Phys. 38, p. 27 (1999).

8. S.W. Lee, S.I. Vlaskina, V.I. Vlaskin, I.V. Zaharchenko, V.A. Gubanov, G.N. Mishinova, G.S. Svechnikov, V.E. Rodionov, S.A. Podlasov, Silicon carbide defects and luminescence centers in current heated 6H-SiC // Semiconductor Physics, Quantum Electronics and Optoelectronics, 13(1), p. 24-29 (2010).

9. S.I. Vlaskina, G.N. Mishinova, L.I. Vlaskin, V.E. Rodionov, G.S.Svechnikov, Nanostructures in lightly doped silicon carbide crystals with polytypic defects // Semiconductor Physics, Quantum Electronics and Optoelectronics, 17(2), p. 155-159 (2014).

10. S. Shinozaki, K.R. Kisman, Aspects of "one dimensional disorder" in silicon carbide // Acta Metallurgica, 26, p. 769-776 (1978).

11. L.U. Ogbuji, T.E. Mitchell, A.H. Heuer, The $\beta \rightarrow \alpha$ transformation in polycrystalline $\mathrm{SiC}$ : The thickening of $\alpha$ plates // J. Amer. Ceram. Soc. 64(2), p. 91-99 (1981).

12. Kozuaki Kobayashi, Shojiro Komatsu. Firstprinciple study of $8 \mathrm{H}-, 10 \mathrm{H}-, 12 \mathrm{H}-\mathrm{ND} 18 \mathrm{H}-\mathrm{SiC}$ polytypes // J. Phys. Soc. Jpn. Appl. 024714 (2012). 